University of Nebraska - Lincoln

DigitalCommons@University of Nebraska - Lincoln

May 1982

\title{
Simple vibrating sample magnetometer
}

\author{
J.A. Gerber \\ University of Nebraska - Lincoln \\ W.L. Burmester \\ University of Nebraska - Lincoln \\ David J. Sellmyer \\ University of Nebraska-Lincoln, dsellmyer@unl.edu
}

Follow this and additional works at: https://digitalcommons.unl.edu/physicssellmyer

Part of the Physics Commons

Gerber, J.A.; Burmester, W.L.; and Sellmyer, David J., "Simple vibrating sample magnetometer" (1982). David Sellmyer Publications. 190.

https://digitalcommons.unl.edu/physicssellmyer/190

This Article is brought to you for free and open access by the Research Papers in Physics and Astronomy at DigitalCommons@University of Nebraska - Lincoln. It has been accepted for inclusion in David Sellmyer Publications by an authorized administrator of DigitalCommons@University of Nebraska - Lincoln. 


\title{
Simple vibrating sample magnetometer
}

\author{
J. A. Gerber, ${ }^{\text {a) }}$ W. L. Burmester, ${ }^{\text {b) }}$ and D. J. Sellmyer \\ Behlen Laboratory of Physics, University of Nebraska, Lincoln, Nebraska 68588 \\ (Received 8 September 1980; accepted for publication 19 January 1982)
}

Design features of a simple and relatively inexpensive homemade vibrating sample magnetometer are described. These include signal electronics which employs an analog divider rather than the usual electromechanical feedback circuit, and a method for gas cleaning during sample changes allowing long experiments at liquid helium temperatures in a superconducting solenoid.

PACS numbers: $07.55 .+\mathrm{x}, 07.20 . \mathrm{Mc}$

\section{INTRODUCTION}

For the past 20 years the vibrating sample magnetometer $(\text { VSM })^{1}$ has found widespread use in both academic research and industry. Popularity of the VSM as a way to measure a sample's magnetic moment as a function of applied field is evidenced by many papers describing the use of this versatile instrument. ${ }^{1-8}$

While many papers deal with mechanical, cryogenic, and pick-up coil-design criteria for implementation of a VSM there is relatively little discussion of the VSM signal recovery electronics, which have often featured an electro-mechanical feedback design or manual signal balancing. In addition there are some mechanical and cryogenic problems that must be surmounted in any homemade VSM. Therefore, the purposes of this note are: (a) to describe two simple and inexpensive methods to arrange the electronics necessary for a VSM, and (b) to describe a gas-cleaning system which ensures long and continuous operation of the VSM at cryogenic temperatures.

\section{ELECTRONICS}

Unlike many of the VSM's described to date we do not use negative feedback schemes to recover the magnitude of the magnetic moment. Feedback circuits are useful because they correct for small changes in VSM drive amplitude and frequency and also give a readout proportional to moment. The circuits described here accomplish the same functions by the use of analog dividers.

To make clear the operation of the analog divider circuits a simple circuit is described which features only electronic instruments readily available in most laboratories. As shown in Fig. 1 an electromechanical driver (voice coil) moves a drive rod with a small vertical amplitude, $A$, and frequency, $\omega$. Mounted on the drive rod is a sample, $S$, and a constant magnitude reference magnetic moment, $R$, which may be a small permanent magnet or small coil with a constant dc excitation current. Pick-up coils near $R$ (see Refs. 2 and 8 for design criteria) will have an induced voltage

$$
V_{R}=k_{R} \omega A \exp (i \omega t) M_{R},
$$

where $k_{R}$ is a constant depending on coil geometry, and $M_{R}$ is the constant magnetic moment of reference $R$. $V_{R}$ is in the millivolt range and easily measured by a rms voltmeter. Similarly, the sample pick-up coils will see a voltage

$$
V_{S}=k_{S} \omega A \exp (i \omega t) M_{S} .
$$

An rms-to-dc conversion is performed on both $V_{R}$ and $V_{S}$ by the rms voltmeter and lock-in amplifier, respectively. ${ }^{9}$ The ratio of these two dc voltages is then taken by a digital multimeter (DMM) which has ratio capabilities. ${ }^{10}$ Taking the root-mean-square average of Eqs. (1) and (2) it is seen that the dependence on frequency, $\omega$, and drive amplitude, $A$, cancel and the DMM output is

$$
V_{\text {out }}=\left\langle V_{s}\right\rangle_{\mathrm{rms}} /\left\langle V_{R}\right\rangle_{\mathrm{rms}}=K M_{S},
$$

where $K$ is the calibration constant which has absorbed $k_{R}, k_{S}$, and $M_{R}$. A calibration potentiometer added to the circuit as shown in Fig. 1 allows the DMM to read directly in units of emu. ${ }^{1}$

The second method to arrange the VSM electronics is identical to that just described, except that the rms voltmeter and ratio mode DMM are replaced by an inexpensive circuit made with readily available integrated circuits. Figure 2 shows the circuit used successfully in our lab for several years. The circuit has three sections: preamplifier, ac-to-dc converter, and divider. $A_{1}$ is a highinput impedance amplifier, $A_{2}$ through $A_{5}$ are all general purpose operational amplifiers. ${ }^{12}$ The reference voltage, $V_{R}$ (Fig. 1), is connected to the preamplifier through terminal $T_{1}$. Preamplifier gain should be set via $R_{6}$ to provide an output at $A_{2}$ of approximately $1 \mathrm{~V}$ peak to peak. Gain of the preamplifier in Fig. 2 is 5000 but in practice will depend on the specific reference coil geometry and reference moment. The amplified ac reference voltage is available at $T_{2}$ for display on an oscilloscope. ${ }^{13}$ The ac-to-dc conversion is done by the circuit incorporating $\mathrm{A}_{3}$ and $\mathrm{A}_{4} \cdot{ }^{14}$ The variable gain provided by $R_{15}$ and $A_{5}$ allows the dividers denominator voltage to be set near $10 \mathrm{~V}$ for maximum divider accuracy. Meter $M_{1}$ is optional. It displays the averaged reference signal for additional visual confirmation that the system is operating properly. The sample signal (lock-in amplifier output) enters the divider circuit (numerator) through terminal $T_{3}$. The divider itself is a monolithic four-quadrant divider ${ }^{15}$ circuit which can handle both positive and negative numerator voltage (parallel or antiparallel moment). The divider output is proportional to the sample's magnetic moment and can be taken from $\mathrm{T}_{4}$ to the $y$ axis 


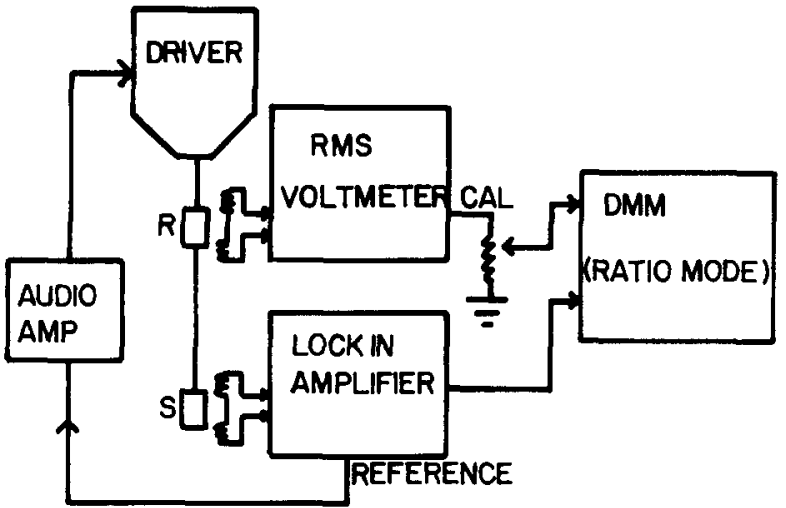

FIG. 1. Block diagram of VSM electronics.

of an $x-y$ recorder. If the $x$ axis is driven by a voltage proportional to the applied magnetic field, the system will trace out the familiar moment vs field curves in all four quadrants.

\section{MECHANICAL AND CRYOGENIC ASPECTS}

The signal recovery electronics described above is appropriate for any VSM. In the remainder of this paper we describe techniques primarily useful for a VSM operating at cryogenic temperatures. The VSM is mounted within a tall (64 in.) Janis Veri Temp double Dewar ${ }^{16}$ which permits low liquid helium consumption and variations of sample temperature in the range $1.5 \mathrm{~K} \leq T$ $\leq 300 \mathrm{~K}$. With the exception of the superconducting solenoid (U) and sample pick-up coils (R), Fig. 3 shows only the VSM components within the inner wall of the insert Dewar.

The electromechanical drive $(G)$ is a Ling model 203 shaker, ${ }^{17}$ a sturdy arrangement with a threaded hole in it for easy coupling to the sample drive rod (P). The sample is vibrated at $35 \mathrm{~Hz}$. To reduce load in the shaker the drive rod rests in a cradle that is suspended by $\mathrm{Be}-$ $\mathrm{Cu}$ springs $(\mathrm{H})$. The shaker and drive rod are coupled via a point contact. This cradle and point-contact method was adopted for suspending and vibrating the sample to ensure that the position and vibration amplitude of the sample remained constant and reproducible. Another method that has been described to surmount such problems is that of Flanders and Doyle, ${ }^{18}$ who used a VSM with a synchronous motor drive.

The reference pick-up coils, $N$, must be placed far enough from the vibrator to prevent excessive in-phase pick-up noise from the vibrator excitation current. For our tall Dewar (and clearance to ceiling) this meant the reference coils had to be mounted within the inner Dewar. In this region large temperature changes $(\Delta T$ $\approx 100 \mathrm{~K}$ ) meant that a constant reference moment could not be provided by a permanent magnet. The reference moment was a coil (M) with a constant $150 \mathrm{~mA}$ excitation current. If the reference coils can be located at room temperature, the best choice for a reference moment is a permanent magnet.

The sample pick-up coils ( $R$ ) are mounted on the superconducting solenoid (U) in the liquid helium bath. Both sample and reference coils are wound according to the criteria suggested by Springford et al. ${ }^{2}$ Additional comments on design are suggested by Foner. ' Our system has a magnetic moment sensitivity of about $10^{-5} \mathrm{emu}$ at zero field and $10^{-4} \mathrm{emu}$ at $80 \mathrm{kOe}$. Temperature is monitored by a carbon glass resistor ${ }^{6}$ and controlled via a capacitance thermometer and a capacitance bridge temperature feedback control. ${ }^{19}$ The sample holder and guide (Q) were machined from Delrin.

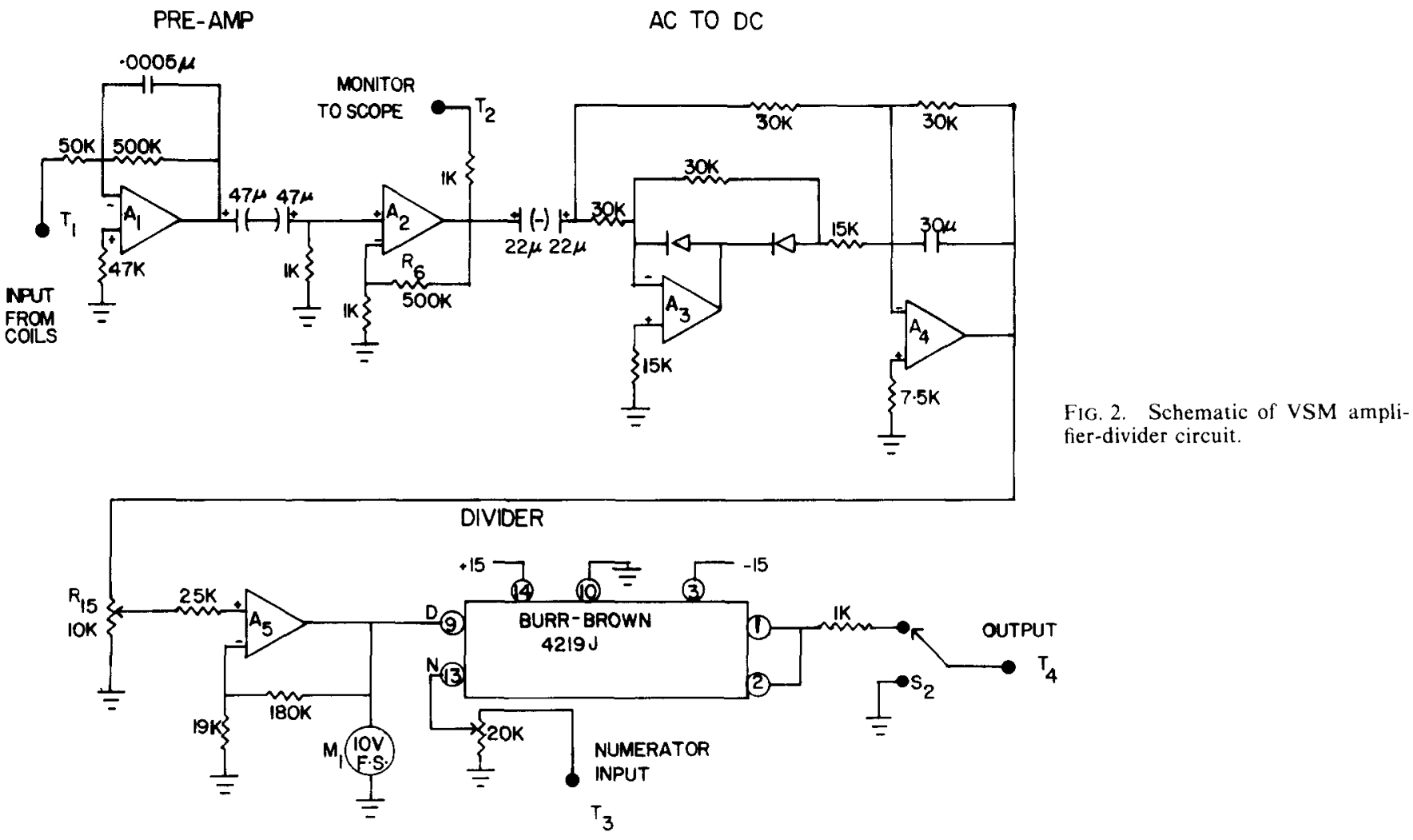




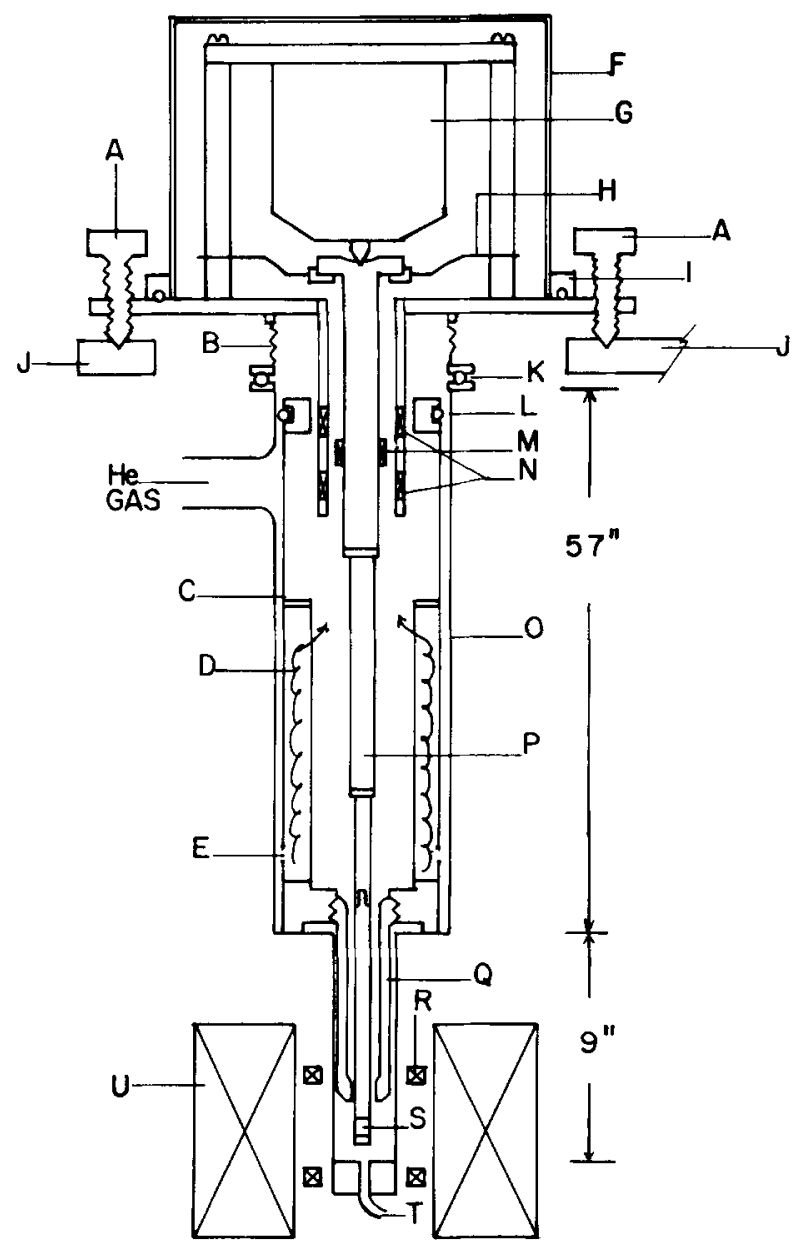

FIG. 3. Cross section of inner Dewar and inserts. (Not to scale) (A) leveling screws, (B) bellows, (C) gas cleaning insert, (D) copper wool, (E) He gas inlet, (F) top hat, (G) electromechanical driver, (H) BeCu spring, (I,K,L) O-ring seals, (J) support bracket (attached to wall and ceiling), (M) reference moment coil, (N) reference pick-up coils, $(\mathrm{O})$ inner wall of insert Dewar, (P) sample drive rod, (Q) sample holder guide, (R) sample pick-up coils, (S) sample, (T) He liquid/gas inlet, (U) superconducting magnet

A particular problem with the VSM described so far (and with some other cryogenic VMS's ${ }^{2}$ ) was the tendency for particles of solid air or water to collect in the lower region of the insert Dewar and prevent smooth movement of the drive rod. The drive rod would inevitably freeze up after about $10 \mathrm{~h}$ of operation (5-8 sample changes) independent of the volume of helium gas used to flush the inner Dewar while samples were being changed. A frozen drive rod forces one to shut down the system and warm it to room temperature. This problem was completely eliminated by the addition of a gas cleaning insert as shown in Fig. 3 [(C), (D), and (E)]. The insert forces incoming helium gas down the wall of the inner Dewar. The gas enters the annular ring at $E$ and then must pass through a copper wool mesh (copper dishscrubbing pads) that fills the annular ring. The cooled and filtered gas then enters the inner Dewar, passes through the pick-up coil support, and exits to the room. The action of this gas system is twofold: (1) any residual water vapor or nitrogen in the helium flush gas (commercial grade bottled helium is used) is trapped in the copper wool and (2), the exiting cold helium gas will condense back-diffusing air or water near the top of the inner Dewar, rather than allowing a collection of frozen debris to collect at the bottom of the Dewar and block the drive-rod motion.

The gas cleaning insert has extended the run time of the VSM and superconducting solenoid by an order of magnitude. Since the installation of the gas cleaner the system has never been shut down due to a frozen drive rod. The VSM is usually operated until the measurements are complete or the experimentors are exhausted. Typically a run lasts seven days.

In summary, we have described several methods that we have found useful in the construction of a simple and relatively inexpensive homemade VSM. Our system has been used successfully to study a variety of magnetic materials including metallic glasses, ${ }^{20}$ low-dimensional compounds, ${ }^{21}$ and intermetallic compounds. ${ }^{22}$

\section{ACKNOWLEDGMENTS}

We are thankful to Dr. F. Szofran and Dr. E. R. Domb for their help in early work on the system, and to the National Science Foundation for support of our research under Grant DMR-7810781 and DMR-8110520.

2) Present address: 3M, 3M Center, Central Research Laboratory, St. Paul, Minnesota 55101

b) Present address: Hughes Research Laboratory, Culver City, California 90230.

${ }^{\prime}$ S. Foner, Rev. Sci. Instrum. 30, 548 (1959).

${ }^{2}$ M. Springford, J. R. Stockton, and W. R. Wampler, J. Phys. E 4, 1036 (1971).

${ }^{3}$ E. E. Bragg and M. S. Seehra, J. Phys. E 9, 216 (1976).

${ }^{4}$ R. P. Guertin and S. Foner, Rev. Sci. Instrum. 45, 863 (1974).

${ }^{5}$ N. F. Oliveira, Jr. and S. Foner, Rev. Sci. Instrum. 43, 37 (1972).

${ }^{6}$ G. J. Bowden, J. Phys. E 5, 1115 (1972).

${ }^{7}$ S. Foner and E. J. McNiff, Jr., Rev. Sci. Instrum. 39, 171 (1968).

${ }^{8}$ S. Foner, Rev. Sci. Instrum. 46, 1425 (1975)

${ }^{9}$ For example: Hewlett Packard \#3400-A RMS Voltmeter, and Princeton Applied Research \#126.

${ }^{10}$ Such a Digital multimeter is the Data Precision model 3500, Data Precision Corp., Danvers, Massachusetts.

"A digital output from the DMM may be connected directly to a computer. Unless the DMM has an analog output (not a common feature) the DMM's ratio result cannot drive the $y$ axis of an $x-y$ recorder. This problem is eliminated when using the circuit of Fig 2.

${ }^{12}$ Our circuit uses RCA \#CA3140 for A1, RCA \#CA741 for A2A5.

${ }^{13}$ We have found that observing the ac reference signal on a general purpose oscilloscope is of great value for monitoring the operation of the VSM. A quick glance at the scope should show a smooth sine wave confirming proper operation. A clipped sine wave, reduced amplitude, harmonic components, or noisy signal indicate overvoltage on the electromechanical driver, undervoltage to the driver, bending modes in the drive rod, or solid air particles causing binding on the sample rod slider, respectively.

${ }^{14} \mathrm{E}$. R. Hnatek, Applications of Linear Integrated Circuits (Wiley, New York, 1975).

${ }^{15}$ Analog Divider \#4291, Burr-Brown Research Corp., Tucson, Arizona.

16 Janis Research, Stoneham, Massachusetts.

${ }^{17}$ Ling Electronics, Anaheim, California.

${ }^{18}$ P. J. Flanders and W. D. Doyle, Rev. Sci. Instrum. 33, 691 (i962).

${ }^{19}$ The capacitance controller is sold by Lake Shore Cryotronics, Eden, New York. See also: H. H. Sample and L. G. Rubin, Cryogenics 17, 594 (1977)

${ }^{20}$ See, for example: G. R. Gruzalski and D. J. Sellmyer, Phys. Rev. B 20, 184 (1979); ibid, 20, 197 (1979); J. A. Gerber, S. G. Cornelison, W. L. Burmester, and D. J. Sellmyer, J. Appl. Phys. 50, 1608 (1979)

${ }^{21}$ S. J. Hillenius, R. V. Coleman, E. R. Domb, and D. J. Sellmyer, Phys. Rev. B 19, 4711 (1979); E. R. Domb, D. J. Sellmyer, and G. D. Sturgeon, J. Phys. Chem. Solids 40, 739 (1979).

${ }^{22}$ T.-H. Tsai and D. J. Sellmyer, Phys. Rev. B 20, 4577 (1979). 